\title{
Design of PIDA Controller Using Bat Algorithm for AVR Power System
}

\author{
D. K. Sambariya*, D. Paliwal \\ Department of Electrical Engineering, Rajasthan Technical University, Kota, 324010, India
}

Copyright (C)2016 by authors, all rights reserved. Authors agree that this article remains permanently

open access under the terms of the Creative Commons Attribution License 4.0 International License

\begin{abstract}
In this article, a new proportional, integral, derivative, and acceleration (PIDA) controller for an automatic voltage regulator power system is presented. The controller parameters are designed by considering as an optimization with minimization of square error using bat algorithm. The response of the bat algorithm optimized PIDA (BA-PIDA) controller are observed and compared to the controllers in the literature. The superiority is validated in terms of performance indices (ITAE, IAE and ISE.
\end{abstract}

Keywords Proportional Integral Derivative Acceleration (PIDA), Bat Algorithm, Current Search Algorithm, Genetic Algorithm, Tabu Search

\section{Introduction}

The application of control system in the design of an efficient and economic controller is always a challenge to control engineers. The different controller structures and design methodologies are always the concerns of researchers. The proportional- integral-derivative (PID) controllers are generally preferred controllers in industrial applications [1]. In many cases, the systems are modeled as a third order. In fact, the traditional motion control system such as an Ac motor with position control is properly modeled as a third order plant without crude approximations [2]. The design of PID controllers is not always easy in some cases. It is the reason that a new structure of controller becomes necessity of such systems [3].

In 1996, Jung and Dorf proposed a new structure of controller and termed as proportional- integral-derivative and acceleration (PIDA) controller [2]. It consists of three numbers of zeros and poles but two poles may be neglected in the design process. It is addition of a zero in the standard PID structure to derive the PIDA structure of the controller.The introduction of an extra zero to the PID controller is to change the root locus of the third order plant in order to make dominant roots more dominant by eliminating the effects of nondominant roots [4].
Initially, the design of PIDA controller parameters have been determined using analytical method. In this method two characteristic equations; one formed with desired root locations with specifications based on the design criterion, and another one with the nominal control structure were equated to deduce the parameters of PIDA. It have been considered to design PIDA controller for third order systems and extended for the control of an AC motor system [2]. The application of PIDA were considered for a servo motor driving a load through a long shaft or transmission system in [5]. Sambariya and Paliwal, 2016 have presented the optimal tuning of PIDA controller parameters using harmony search algorithm in [6].

Dal-Young et. al, 2001, highlighted the higher overshot during the rise time while designed PIDA controller with analytical method. It were mitigated using pre-compensator to PIDA in ac motor control in [7]. The performance of PIDA controller for the third order system have been improved using Kitt's method and extended to discrete system [8, 9]. The design of PIDA for third order systems were presented using an algebraic design approach which also utilized the genetic algorithm (GA) to achieve design optimality in [10].

The recent current search algorithm have been proposed to determine the PIDA parameters for automatic voltage regulator (AVR) system in [4]. It have compared the system response with PIDA controller using genetic algorithm (GA) and tabu search (TS) algorithm and proved to be superior with current search algorithm. In this paper, the PIDA controller parameters are designed using bat algorithm.

The article is organized with five sections. The detail on the problem formulation is given in section 2 . The bat algorithm is described in section 3. The response of the AVR system with bat algorithm based PIDA controller is compared to response that of with controllers in [4] in section 4. Finally, the article is concluded in section 5 and followed by references. 


\section{Problem formulation}

\subsection{AVR System}

The application of an automatic voltage regulator (AVR) in the generator excitation system to control the reactive power flow and regulate the generator voltage was presented in [11, 12]. It holds the terminal voltage of a generator at a specified level. It critically affect the security of the power system [3].

The AVR consists of amplifier, exciter, generator, and sensor. A simplified AVR system controlled by the PIDA controller is represented by the block diagram in Fig. 1, where $V_{e}$ is the error voltage between the reference input voltage $V_{\text {ref }}(s)$ and sensor voltage, while $V_{u}, V_{r}$, and $V_{f}$ are the controlled, amplified, and excited voltage signals, and $V_{t}(s)$ is the output voltage. The detail on the linearized AVR system is mentioned as following [4]:

\subsubsection{Amplifier model}

The amplifier model in AVR system is expressed in Eqn. 1. The gain and time constants are presented by $K_{A}$ and $\tau_{A}$, respectively. The recommended values of $K_{A}$ and $\tau_{A}$ are in the range of 10 to 400 and from 0.02 to 0.1 seconds, respectively [13]. The considered values of these parameters is as $K_{A}=10$ and $\tau_{A}=0.1$ seconds.

$$
\frac{V_{r}(s)}{V_{u}(s)}=\frac{K_{A}}{\tau_{A} s+1}
$$

\subsubsection{Exciter model}

The exciter model transfer function is presented in Eqn. 2. The range of gain $K_{E}$ and a time constant $\tau_{e}$ is 1 to 400 and 0.25 to 1.0 seconds, respectively $[13,11]$. The selected values of these parameters are $K_{E}=1$ and $\tau_{e}=0.4$ seconds.

$$
\frac{V_{f}(s)}{V_{r}(s)}=\frac{K_{E}}{\tau_{E} s+1}
$$

\subsubsection{Generator model}

The transfer function representation of generator model in AVR system is given in Eqn. 3. It consists with a gain $K_{G}$ and the time constant $\tau_{G}$. The range may vary between 0.7 to 1.0, and 1.0 to 2.0 seconds, respectively [13]. The considered values are 1 and 1 second, respectively.

$$
\frac{V_{t}(s)}{V_{f}(s)}=\frac{K_{G}}{\tau_{G} s+1}
$$

\subsubsection{Sensor model}

The sensor model is considered as a simple first-order transfer function as stated in Eqn.4. The gain $K_{R}$, and the time constant $\tau_{G}$ in the range from of 1 to 10 and 0.001 to 0.06 second, respectively [13]. In this work, $K_{R}=1$ and $\tau_{G}$ $=0.01$ second are considered.

$$
\frac{V_{s}(s)}{V_{t}(s)}=\frac{K_{R}}{\tau_{R} s+1}
$$

\subsection{PIDA Controller}

The transfer function representation of the PIDA controller is expressed in Eqn. 5. The terms $K_{p}, K_{i}, K_{d}$, and $K_{a}$ in this controller are the proportional, integral, derivative, and accelerated gains, respectively, while $d$, and $e$ are filter parameters. The transfer function in Eqn. 5, is alternative presented in a polynomial form in Eqn. 6 with the parameters $K_{p}, K_{i}, K_{d}$, $K_{a}, \alpha$, and $\beta$, respectively [4].

$$
\begin{gathered}
G_{c}(s)=K_{p}+\frac{K_{i}}{s}+\frac{K_{d} s}{s+d}+\frac{K_{a} s^{2}}{(s+d)(s+e)} \\
G_{c}(s)=\frac{K_{a} s^{3}+K_{d} s^{2}+K_{p} s+K_{i}}{s^{3}+\alpha s^{2}+\beta s}
\end{gathered}
$$

\subsection{Objective function}

In this article, the design of PIDA controller is carried out considering the problem as optimization. The objective function during optimization is considered as in Eqn. 7 with parameter bounds as in Eqn. 8. The optimization is considered with minimization of error signal as in Eqn. 7.

Minimize:

$$
J=\int_{t=0}^{T_{s i m}}\left|V_{r e f}(t)-V_{t}(t)\right|^{2} d t
$$

Subjected:

$$
\begin{aligned}
& K_{a}^{\min } \leq K_{a} \leq K_{a}^{\max } \\
& K_{d}^{\min } \leq K_{d} \leq K_{d}^{\max } \\
& K_{p}^{\min } \leq K_{p} \leq K_{p}^{\max } \\
& K_{i}^{\min } \leq K_{i} \leq K_{i}^{\max } \\
& \alpha^{\min } \leq \alpha \leq \alpha^{\max } \\
& \beta^{\min } \leq \beta \leq \beta^{\max }
\end{aligned}
$$

\section{Proposed Bat Algorithms}

The bat algorithm is based on the echolocation behaviour of Micro bats [14]. In echolocation, each pulse generated by a micro bat may last only for 8 to 10 milliseconds with a frequency ranging from $25 \mathrm{kHz}$ to $150 \mathrm{kHz}$, which corresponds to the wavelengths of $2 \mathrm{~mm}$ to $14 \mathrm{~mm} \mathrm{[15].} \mathrm{In} \mathrm{BA,}$ the echolocation characteristics of Micro bats can be idealized with the following assumptions.

- It is assumed that the bats are able to detect distance of prey, background obstacles and difference in the available prey/food in the search path in some magical way using echolocation property [16].

- An $k^{t h}$ Bat may randomly fly with location as $x_{k}$, velocity as $v_{k}$, frequency as $f_{\text {min }}$ but with varying wavelength as $\lambda_{k}$ and loudness of echo as $A^{0}$ to search food/prey. The Micro bats have an ability to adjust frequency (wavelength) of the emitted pulses of echo and rate of pulse emission out of $r \in[0,1]$ according to the distance of their prey/food [17].

- The loudness of the echo pulse should be varied as reducing with decreased distance of the food, i.e. from large $A^{0}$ to a minimum value $A_{\min }$ (at target/prey location) [18]. 


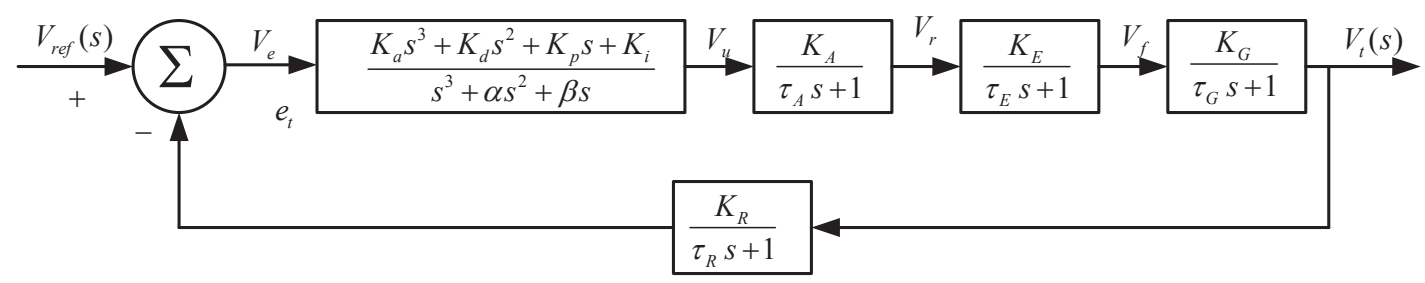

Figure 1. Representation of closed loop AVR system with PIDA controller

\subsection{Procedural Steps}

Let in an optimization problem; the objective function be represented by Minimization of $F(x)$ which is subjected to $x_{k} \in X_{k}, k=1,2,3, \ldots, N[19]$.

\subsubsection{Initialization}

- As an initial step, the bat population is initiated as position and velocity as $v_{k}$ with $k=1,2,3, \ldots, n$.

- Initial pulse frequency is defined as $f_{k} \in\left[f_{\min }, f_{\max }\right]$.

- The pulse rates $r_{k}$ and the loudness $A_{k}$ are also set as above

- Check number of iterations or $t<T_{\max }$

\subsubsection{Generation of new solutions}

- New solutions may be generated by adjusting the pulse frequency and keeping wavelength as constant.

- For each bat $(k)$, its position $x_{k}$ and velocity $v_{k}$ in a d-dimensional search space should be defined. $x_{k}$ and $v_{k}$ should be subsequently updated during the iterations $[20,21]$. The new solutions $x_{k}^{t}$ and velocities $v_{k}^{t}$ at time step $t$ can be calculated by:

$$
\begin{gathered}
f_{k}=f_{\min }+\left(f_{\max }-f_{\min }\right) \beta \\
v_{k}^{t}=v_{k}^{t-1}+\left(x_{k}^{t-1}-x^{\prime}\right) f_{k} \\
x_{k}^{t}=x_{k}^{t-1}+v_{k}^{t}
\end{gathered}
$$

where, $\beta$ is defined for uniform distribution as a vector and selected as $\beta \in[0,1]$. The $x^{\prime}$ stands as the best location in search space after comparing solutions of all the $n$ bats [22]. The product of $f_{k}$ and $\lambda_{k}$ represents the velocity increment. The velocity increment can be adjusted by changing one and keeping fixed another according to a problem. The generally used range of frequency is $0 \leq f \leq 100$ and each bat at initialization step is selected from $f=\left[f_{\min }, f_{\max }\right][23,24]$.

\subsubsection{Local Search}

Once the best current solution is selected among the available solutions, then a new solution is generated by using local random walk and assigned to each bat as in Eqn. 12 . If $\varepsilon \in[-1,1]$ represents a random number range and $A^{t}=\left\langle A_{k}^{t}\right\rangle$ stands for average value of loudness of all initiated $n$ bats at time $t$.

$$
x_{n e w}=x_{o l d}+\varepsilon A^{t}
$$

\subsubsection{Bat flying and Generation of a new solutions}

As the number of iteration increases, the loudness $A_{k}$ and the rate $r_{k}$ of pulse emission have to be updated. As a microbat reaches to its target/prey the rate of pulse emission increases while the loudness decreases. The loudness is generally selected from $\left[A^{0}, A_{\text {min }}\right]=[1,0]$. The $A^{0}=1$ represents the maximum loudness of emitted pulse by microbat in search of prey, while $A_{\min }=0$ indicates that the microbat got the target/prey and not emitting any loudness. Thus, the loudness and the rate of pulse emission is updated as.

$$
\begin{gathered}
A_{k}^{t+1}=\alpha A_{k}^{t} \\
r_{k}^{t+1}=r_{k}^{0}\left[1-e^{-\gamma t}\right]
\end{gathered}
$$

where, $\alpha$ and $\gamma$ represent the constant values. Here, $\alpha$ is similar to the cooling factor of a cooling schedule in the simulated annealing [25] and the range of these constants is as $0<\alpha<1$ and $0<\gamma$.

$$
\begin{aligned}
& A_{k}^{t} \rightarrow 0 \\
& r_{k}^{t} \rightarrow r_{k}^{0}
\end{aligned}
$$

To make optimization simpler, the value of $\alpha$ and $\gamma$ should be selected as same, therefore, in this study $\alpha=\gamma=0.9$. As in Eqns. 15 - 16, the initial loudness and emission rate may be represented by $A_{k}^{0}$ and $r_{k}^{0}$, respectively. The value of emission rate at time $t$ can be selected from $r_{k}^{0} \in[0,1]$.

\subsubsection{Checking the stopping criterion}

If the maximum count of iterations is reached as a stopping criterion is satisfied, then the process of computation is terminated. Otherwise, go to steps 3 and 4 to repeat the process.

\section{Results and discussion}

The PIDA controller for AVR system is designed using bat algorithm (BA). The performance of BA and the BA-PIDA controller is designed in the next sections.

\subsection{Performance of bat algorithm}

The scheme of optimization of PIDA controller using BA with an ISE based objective function is considered and shown in Fig. 3. The initializing parameters for bat algorithm are as mentioned following and the lower and upper bounds for PIDA parameters are considered as in Eqn. 17.

- Population size, typically 10 to 40 and considered as 20 


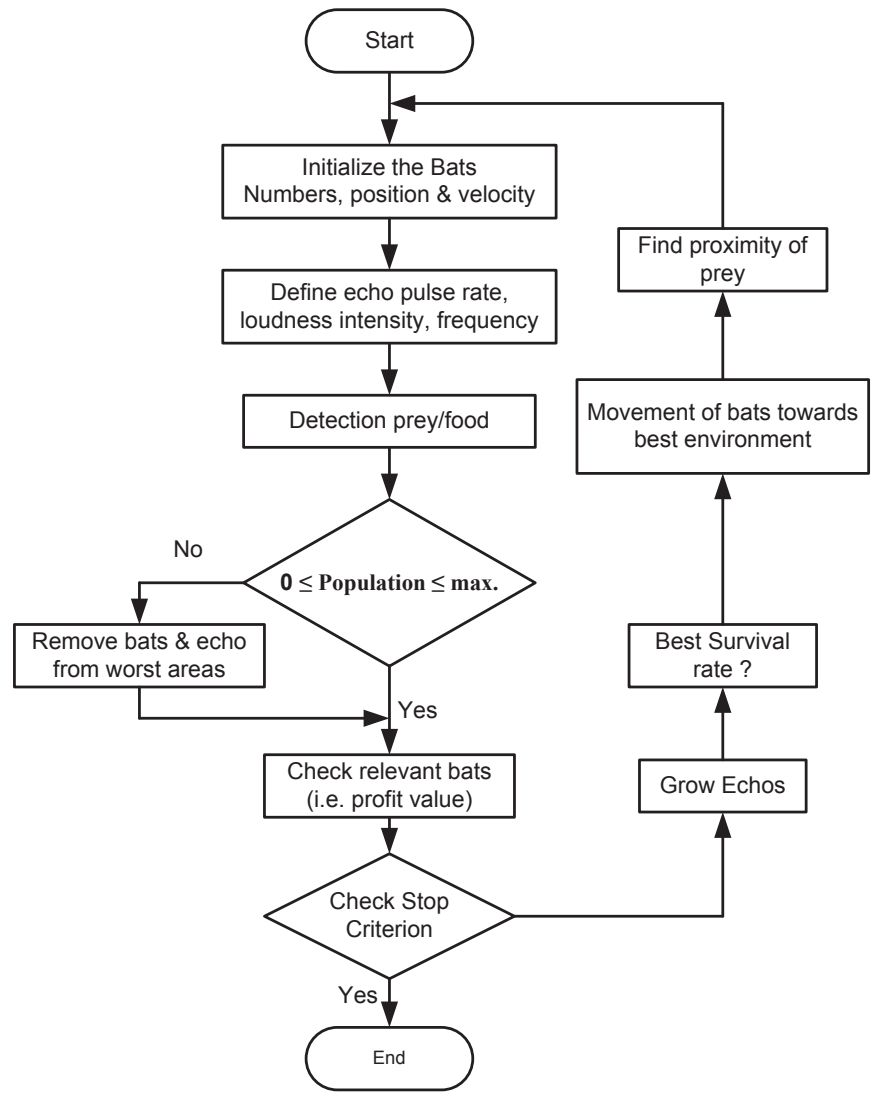

Figure 2. Working of bat Algorithm

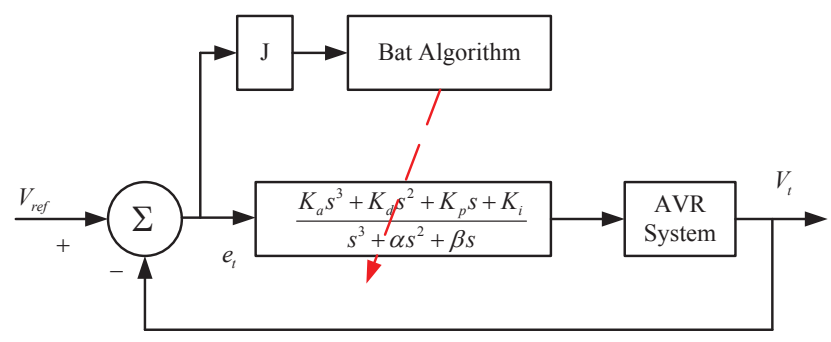

Figure 3. Representation of optimization scheme of the PIDA controller using bat algorithm

- Number of generations selected as 100

- Loudness (constant or decreasing) selected as 0.5

- Pulse rate (constant or decreasing) selected as 0.5

- Frequency minimum selected as 0.01

- Frequency maximum selected as 2

- Number of dimensions selected as 6

$$
\begin{aligned}
& 50 \leq K_{a} \leq 150 \\
& 450 \leq K_{d} \leq 550 \\
& 750 \leq K_{p} \leq 850 \\
& 350 \leq K_{i} \leq 450 \\
& 550 \leq \alpha \leq 650 \\
& 900 \leq \beta \leq 1000
\end{aligned}
$$

The performance of bat algorithm in terms of fitness function and iterations is shown in Fig. 4. The unknown parameters of the PIDA controller are recorded at the end of
Table 2. Comparison of system response in terms of Performance indices

\begin{tabular}{lccc}
\hline Controller & ITAE & IAE & ISE \\
\hline PIDA-BA (Proposed) & 199.79 & 19.724 & 19.585 \\
PIDA-CS [4] & 199.97 & 19.765 & 19.697 \\
PIDA-GA [4] & 199.90 & 19.743 & 19.650 \\
PIDA-TS [4] & 199.89 & 19.760 & 19.680 \\
\hline
\end{tabular}

iteration 100, where, the process of optimization get terminated. These parameters are recorded in Table 1. It also enlist the parameters with genetic algorithm (GA), tabu search (TS) and current search (CS) as reported in [4].

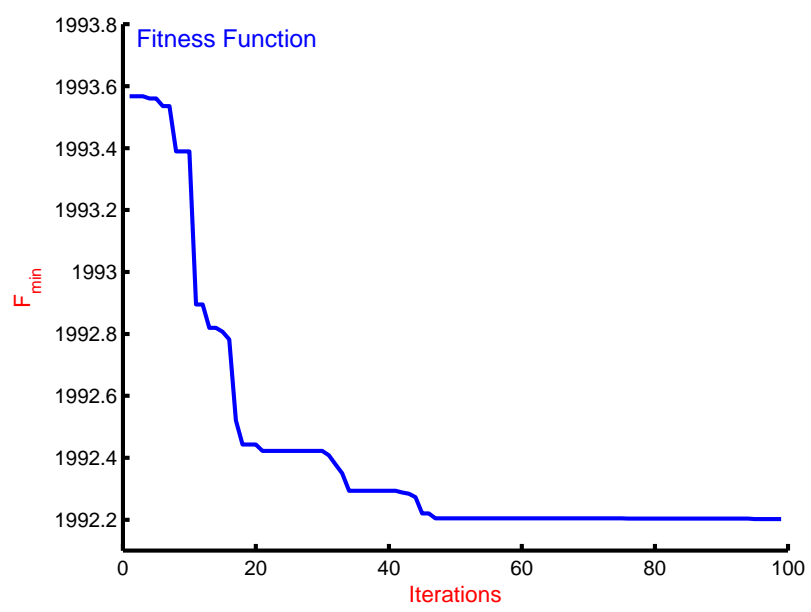

Figure 4. Representation of fitness function using bat algorithm

\subsection{Simulation results}

The simulation study of the AVR power system is caried out without controller and with PIDA controllers. Initially, the system without controller is simulated and the response is shown in Fig. 5. The AVR system is equipped with PIDA controller with parameters in Table 1. The responses are observed and compared as shown in Fig. 6. It is clear from the figure that the response with proposed BA-PIDA outperforms as presented in [4]. The critical analysis was carried out in terms of performance indices. These performance indices are integral time weighted error (ITAE), integral absolute error (IAE) and integral of squared error (ISE) as defined by Eqn. 18 - Eqn. 20, respectively. The detail on performance indices may be referred as in [26, 27, 28, 1, 29]. The observed performance indices are enlisted in Table 2. The ITAE value with proposed PIDA-BA controller is 199.82 which is lesser as compared to 199.97. 199.90 and 199.89 with controllers in [4]. It means that the proposed PIDA-BA outperforms the PIDA-CS, PIDA-GA and PIDA-TS.

$$
\begin{aligned}
I T A E & =\int_{0}^{T_{s i m}} t\left|y_{o}(t)-y_{r}(t)\right| d t \\
I A E & =\int_{0}^{T_{s i m}}\left|y_{o}(t)-y_{r}(t)\right| d t
\end{aligned}
$$


Table 1. Comparison of the PIDA parameters

\begin{tabular}{lcccccc}
\hline Controller & $K_{a}$ & $K_{d}$ & $K_{p}$ & $K_{i}$ & $\alpha$ & $\beta$ \\
\hline PIDA-BA (Proposed) & 149.9821 & 549.5267 & 755.1120 & 350.0000 & 550.2274 & 1000.000 \\
PIDA-CS [4] & 101.0102 & 498.9516 & 799.1201 & 394.0112 & 589.5411 & 965.0501 \\
PIDA-GA [4] & 99.1016 & 577.5625 & 784.0012 & 342.1474 & 592.9654 & 912.4471 \\
PIDA-TS [4] & 102.2365 & 592.3258 & 824.8747 & 341.6321 & 596.1974 & 854.1247 \\
\hline
\end{tabular}

$$
I S E=\int_{0}^{T_{\text {sim }}}\left|y_{o}(t)-y_{r}(t)\right|^{2} d t
$$

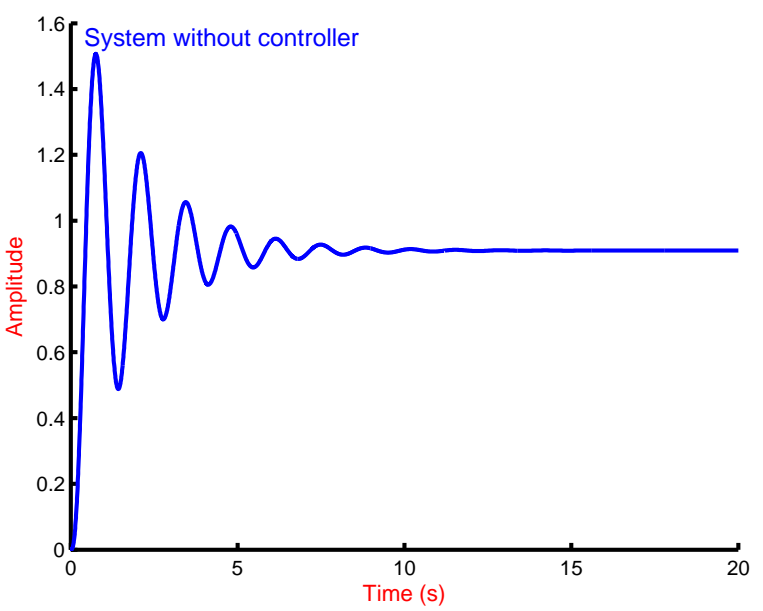

Figure 5. Step response of the AVR system without controller

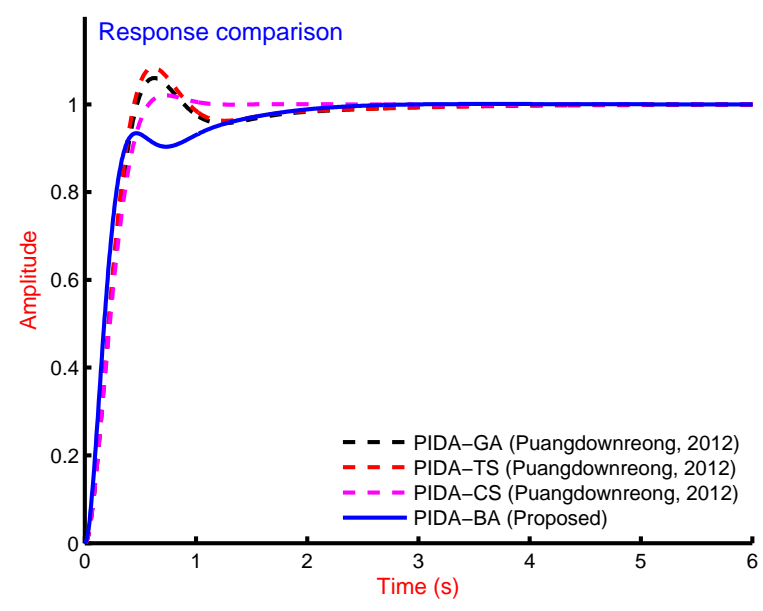

Figure 6. Step response of the AVR system with controllers

\section{Conclusion}

The optimum PIDA controller is obtained using bat algorithm. The performance of this controller is compared to that of with current search, genetic algorithm and tabu search algorithms and outperforms these. The effectiveness of the proposed BA-PIDA is presented graphically and in terms of performance indices (ITAE, IAE and ISE).

\section{REFERENCES}

[1] D. K. Sambariya and R. Prasad, "Design of robust PID power system stabilizer for multimachine power system using HS algorithm," American Journal of Electrical and Electronic Engineering, vol. 3, no. 3, pp. 75-82, 2015. [Online]. Available: http://dx.doi.org/10.12691/ajeee-33-3

[2] S. Jung and R. Dorf, "Analytic pida controller design technique for a third order system," in Decision and Control, 1996., Proceedings of the 35th IEEE Conference on, vol. 3, Dec 1996, pp. 2513-2518 vol.3. [Online]. Available: http://dx.doi.org/10.1109/CDC.1996.573472

[3] D. K. Sambariya and V. Nath, "Optimal control of automatic generation with automatic voltage regulator using particle swarm optimization," Universal Journal of Control and Automation, vol. 3, no. 4, pp. 63-71, 2015. [Online]. Available: http://dx.doi.org/10.13189/ujca.2015.030401

[4] D. Puangdownreong, "Application of current search to optimum pida controller design," Intelligent Control and Automation, vol. 3, no. 0, pp. 303-312, 2012. [Online]. Available: http://dx.doi.org/10.4236/ica.2012.34035

[5] C. U-thaiwasin, S. Sujitjorn, Y. Prempraneerach, and J. Ngamwiwit, "Torsional resonance suppression via pida controller," in TENCON 2000. Proceedings, vol. 3, Sept 2000, pp. 498-503 vol.2. [Online]. Available: http://dx.doi.org/10.1109/TENCON.2000.892316

[6] D. K. Sambariya and D. Paliwal, "Optimal design of pida controller using harmony search algorithm for avr power system," in 6th IEEE International Conference on Power Systems, (ICPS-2016), 2016, pp. $1-6$.

[7] H. Dal-Young, L. Ihn-Yong, C. Young-Seung, L. Young-Do, and C. Boo-Kwi, "The design of pida controller with precompensator [for induction motors]," in Industrial Electronics, 2001. Proceedings. ISIE 2001. IEEE International Symposium on, vol. 2, 2001, pp. 798-804 vol.2. [Online]. Available: http://dx.doi.org/10.1109/ISIE.2001.931570

[8] P. Ukakimaparn, P. Pannil, P. Boonchuay, and T. Trisuwannawat, "PIDA controller designed by kitti's method," in ICCAS-SICE, 2009, Aug 2009, pp. 1547-1550.

[9] K. Smerpitak, P. Ukakimaparn, T. Trisuwannawat, and P. La-orsri, "Discrete-time PIDA controller designed by kitti's method with bilinear transform," in Control, Automation and Systems (ICCAS), 2012 12th International Conference on, 2012, pp. 1585-1590.

[10] S. Sornmuang and S. Sujitjorn, "Ga-based optimal PIDA controller design," in Proceedings of the 10th WSEAS international conference on Systems theory and scientific computation. World Scientific and Engineering Academy and Society (WSEAS), 2010, pp. 192-197.

[11] V. Nath and D. K. Sambariya, "Analysis of AGC and AVR for single area and double area power system using fuzzy logic control," International Journal of Advanced Research in Electrical, Electronics and Instrumentation Engineering, vol. 4, no. 7, pp. 6501-6511, 2015.

[12] D. K. Sambariya and V. Nath, "Load frequency control using fuzzy logic based controller for multi-area power system," British Journal of Mathematics \& Computer Science, vol. 13, no. 5, pp. xx-xx, 2016. [Online]. Available: http://dx.doi.org/10.9734/BJMCS/2016/22899

[13] Z.-L. Gaing, "A particle swarm optimization approach for optimum design of pid controller in avr system," Energy Conversion, IEEE Transactions on, vol. 19, no. 2, pp. 384-391, June 2004. [Online]. Available: http://dx.doi.org/10.1109/TEC.2003.821821 
[14] X.-S. Yang, "A new metaheuristic bat-inspired algorithm," in Nature Inspired Cooperative Strategies for Optimization (NICSO 2010), ser. Studies in Computational Intelligence, J. Gonzlez, D. Pelta, C. Cruz, G. Terrazas, and N. Krasnogor, Eds. Springer Berlin Heidelberg, 2010, vol. 284, pp. 65-74. [Online]. Available: http://dx.doi.org/10.1007/978-3-642-12538-6_6

[15] D. K. Sambariya and R. Prasad, "Small signal stability enhancement by optimally tuned conventional power system stabilizer using bat algorithm," in International Conference on Advances in Power Generation from Renewable Energy Sources, pp. 366-380.

[16] D. K. Sambariya, R. Gupta, and R. Prasad, "Design of optima input-output scaling factors based fuzzy PSS using bat algorithm," Engineering Science and Technology, an International Journal, vol. xx, no. xx, pp. xx - xx, 2016, in Press. [Online]. Available: http://www.sciencedirect.com/science/article/pii/S2215098615301142

[17] I. Fister, S. Fong, and J. Brest, "A novel hybrid self-adaptive bat algorithm," The Scientific World Journal, vol. 2014, p. 12, 2014. [Online]. Available: http://dx.doi.org/10.1155/2014/709738

[18] D. K. Sambariya and H. Manohar, "Model order reduction by integral squared error minimization using bat algorithm," in IEEE Proceedings of 2015 RAECS UIET Panjab University Chandigarh $21-22^{\text {nd }}$ December 2015, 2015, pp. 1-7.

[19] D. K. Sambariya and R. Prasad, "Application of bat algorithm to optimize scaling factors of fuzzy logic-based power system stabilizer for multimachine power system," International Journal of Nonlinear Sciences and Numerical Simulation, vol. 17, no. 1, pp. 41 - 53, 2016. [Online]. Available: http://dx.doi.org/10.1515/ijnsns-2015-0025

[20] X.-B. Meng, X. Z. Gao, Y. Liu, and H. Zhang, "A novel bat algorithm with habitat selection and doppler effect in echoes for optimization," Expert Systems with Applications, vol. 42, no. 1718, pp. 6350-6364, 2015. [Online]. Available: http://www.sciencedirect.com/science/article/pii/S0957417415002560

[21] A. H. Gandomi and X.-S. Yang, "Chaotic bat algorithm," Journal of Computational Science, vol. 5, no. 2, pp. 224-232, 2014. [Online]. Available: http://www.sciencedirect.com/science/article/pii/S1877750313001099

[22] S. Yilmaz and E. U. Kucuksille, "A new modification approach on bat algorithm for solving optimization problems," Applied Soft Computing, vol. 28, pp. 259-275, 2015. [Online]. Available: http://www.sciencedirect.com/science/article/pii/S1568494614005912

[23] D. K. Sambariya and R. Gupta, "Effective PID-PSS design using bat algorithm for SMIB power system," in 6th IEEE International Conference on Power Systems, (ICPS-2016), 2016, pp. 1-6.

[24] I. J. Fister, D. Fister, and X.-S. Yang, "A hybrid bat algorithm," Neural and Evolutionary Computing, Elektrotehniski vestnik, Electrotechnical review, 2013, p. in press., 2013.

[25] S. Kirkpatrick, C. Gelatt, and M. P. Vecchi, "Optimization by simulated annealing,” Science, vol. 220, no. 4598, pp. 671-680, 1983.

[26] D. K. Sambariya and R. Prasad, "Robust tuning of power system stabilizer for small signal stability enhancement using metaheuristic bat algorithm," International Journal of Electrical Power \& Energy Systems, vol. 61, no. 0, pp. 229 - 238, 2014. [Online]. Available: http://www.sciencedirect.com/science/article/pii/S0142061514001616

[27] _ - "Optimal tuning of fuzzy logic power system stabilizer using harmony search algorithm," International Journal of Fuzzy Systems, vol. 17, no. 3, pp. 457-470, 2015. [Online]. Available: http://dx.doi.org/10.1007/s40815-015-0041-4

[28] D. K. Sambariya, "Power system stabilizer design using compressed rule base of fuzzy logic controller," Journal of Electrical and Electronic Engineering, vol. 3, no. 3, pp. 52-64, 2015. [Online]. Available: http://dx.doi.org/10.11648/j.jeee.20150303.16

[29] D. K. Sambariya and R. Prasad, "Evaluation of interval type-2 fuzzy membership function \& robust design of power system stabilizer for smib power system," Sylwan Journal, vol. 158, no. 5, pp. 289-307, 2014. [Online]. Available: http://sylwan.ibles.org 